\title{
Third Order Nonlinear Optical Susceptibility of Crystalline Oxide Yttria-Stabilized Zirconia
}

\author{
Guillaume MarcaudiD ${ }^{+,}, 1,{ }^{*}$ Samuel Serna, ${ }^{+, 1,2}$ Karamanis \\ Panaghiotis, ${ }^{+, 3}$ Carlos Alonso-Ramos, ${ }^{1}$ Xavier le Roux, ${ }^{1}$ \\ Mathias Berciano, ${ }^{1}$ Thomas Maroutian, ${ }^{1}$ Guillaume Agnus, ${ }^{1}$ \\ Pascal Aubert, ${ }^{1}$ Arnaud Jollivet, ${ }^{1}$ Alicia Ruiz-Caridad, ${ }^{1}$ \\ Ludovic Largeau, ${ }^{1}$ Nathalie ISAC, ${ }^{1}$ Eric Cassan, ${ }^{1}$ Sylvia \\ Matzen, ${ }^{1}$ Nicolas Dubreuil, ${ }^{4,5}$ Michel Rérat ${ }^{\mathbb{D}},{ }^{3}$ Philippe \\ LECOEUR, ${ }^{1}$ AND LAURENT VIVIEN ${ }^{1},{ }^{*}$ \\ + Contributed equally \\ ${ }^{1}$ Centre de Nanosciences et Nanotechnologies (C2N), Université-Paris-Sud, CNRS UMR 9001, Université \\ Paris-Saclay, Orsay 91405, France. \\ ${ }^{2}$ Present address: Department of Materials Science and Engineering, Massachussetts Institute of \\ Technology, Cambridge, MA 02139, USA and Department of Physics, Bridgewater State University, \\ Bridgewater, MA 02325, USA. \\ ${ }^{3}$ Institut des Sciences Analytiques et de Physico-Chimie pour l'Environnement et les Matériaux, CNRS, \\ Université de Pau et des Pays de l'Adour, Technopôle Hélioparc, 2 avenue P. Angot, 64053 Pau Cedex, \\ France \\ ${ }^{4}$ Laboratoire Charles Fabry, Institut d'Optique Graduate School, CNRS, Université Paris-Saclay, 91127 \\ Palaiseau Cedex, France \\ ${ }^{5}$ Present address: LP2N, Institut d'Optique Graduate School, CNRS, Univ. Bordeaux, 33400 Talence, \\ France \\ *guillaume.marcaud@c2n.upsaclay.fr,laurent.vivien@c2n.upsaclay.fr
}

\begin{abstract}
Nonlinear all-optical technology is an ultimate route for next generation ultra-fast signal processing of optical communication system. New nonlinear functionalities need to be implemented in photonics and complex oxides are considered as promising candidates due to their wide panel of attributes. In this context, Yttria-Stabilized Zirconia (YSZ) stands out thanks to its capability to be epitaxially grown on silicon, adapting the lattice for the crystalline oxides family of materials. We report for the first time a detailed theoretical and experimental study about the third order nonlinear susceptibility in crystalline YSZ. Via self-phase modulation induced broadening and considering the in-plane orientation of YSZ, we experimentally obtained an effective Kerr coefficient of $\widehat{n}_{2}^{Y S Z}=4.0 \pm 2 \cdot 10^{-19} \mathrm{~m}^{2} \mathrm{~W}^{-1}$ in a $8 \% \mathrm{~mol}$. YSZ waveguide. In agreement with the theoretically predicted $\widehat{n}_{2}^{Y S Z}=1.3 \cdot 10^{-19} \mathrm{~m}^{2} \mathrm{~W}^{-1}$, the third order nonlinear coefficient of YSZ is comparable to the one of silicon nitride ( $\mathrm{SiN})$, already used in nonlinear optics. These promising results are a new step towards the implementation of functional oxides for nonlinear optical applications.

(C) 2019 Chinese Laser Press
\end{abstract}

\section{Introduction}

Integrated nonlinear photonics has reached the point of meeting some demand of on-chip optical processing and transmission, owing to the CMOS compatibility. Silicon in particular presents the ability of strong optical confinement. Nevertheless, some properties of silicon are not adequate for integrated photonics. Especially, it presents a large two-photon absorption (TPA) in the nearinfrared while it is accompanied by a large third-order nonlinear susceptibility. This condition limits the use of silicon for devices exploiting Raman lasing, [1] Brillouin amplification [2] 
or parametric amplification. [3] In this context, the quest for other materials, as compatible as possible with silicon foundries started several years ago.

In these new plethora of options, promising results have been obtained in silicon nitride, [4] amorphous silicon [5] or arsenic free chalcogenides [6] exploiting third order nonlinearities. More broadly, strongly-correlated materials like crystalline oxides have emerged as a promising family for their extremely large variety of properties from ferroelectricity, optical nonlinearities to ferromagnetism or phase transition. The combination of their tunable properties leads to the development of innovative devices with manifold functionalities in many fields such as solar cells, [7] sensors, [8] electronics [9] or optics [10] to name a few. However, optical nonlinearities are still unknown for the most part of crystalline oxides.

Among the crystalline oxides, Yttria-Stabilized Zirconia (YSZ) is known for its role as a buffer layer for the integration of $\mathrm{LiNbO}_{3}, \mathrm{PbTiO}_{3}, \mathrm{~Pb}(\mathrm{Zr}, \mathrm{Ti}) \mathrm{O}_{3}$ and $\mathrm{YBa}_{2} \mathrm{Cu}_{3} \mathrm{O}_{7}$ thin films on silicon [11-14] and as a solid electrolyte due to its high ionic conductivity. [15] In addition, YSZ exhibits extraordinary thermal, mechanical and chemical stability [16-18] as well as high refractive index about 2.15 in telecom wavelength range and a wide transparency window from UV to Mid-IR. [16,19-21]

Thanks to the development of growth technique, YSZ has recently demonstrated its potential as a new material for low-loss optical waveguides and as a flexible substrate for infrared nano-optics. [22, 23] However, nonlinear optical properties of YSZ have never been investigated.

We present in this work a theoretical study of the third order nonlinearities of YSZ and its experimental characterization. First-principle calculations have been performed to understand the concentration and doping distribution dependence of the third order nonlinearity in YSZ. Then, we have grown high quality YSZ thin films and fabricated low loss waveguide with an optimized material composition. Finally, thanks to a sensitive technique described in details in the reference, [24] we characterized the optical nonlinearity of YSZ. The experimental results presented in this paper are in agreement with calculation and provide a strong first step for the promising integration of crystalline oxide in silicon photonics platform.

\section{First-principle calculations}

Assuming that the optical nonlinearities of YSZ should stem from the intrinsic Nonlinear optical (NLO) properties of the doping substrate, namely, the cubic phase of $\mathrm{ZrO}_{2}\left(\mathrm{c}-\mathrm{ZrO}_{2}\right)$ we proceeded as follows. First, we focused on the static non-linear optical properties of pure c- $\mathrm{ZrO}_{2}$ aiming at a reliable estimation of the order of magnitude of its third order NLO responses in idealized conditions to be used as a guide for our qualitative conclusions. Since little is known about the true local atomistic structure of YSZ, to circumvent the challenging task [25, 26] of determining the most stable local crystal structure of each system considered, the second step comprised computations addressing the importance of the vacancy/dopant distribution on the third order susceptibilities. For this task, we chose two doping concentrations of 3.2 and $33 \% \mathrm{~mol}$. in $\mathrm{Y}_{2} \mathrm{O}_{3}$ representing the dilute limit and a relatively high doping concentration, respectively. Finally, the third and last step of the current theoretical investigation involved simulations on YSZ of increasing concentrations in $\mathrm{Y}_{2} \mathrm{O}_{3}$.

In this work the relative dielectric $\epsilon$ matrix and nonlinear $\chi^{(n)}$ susceptibility tensors were obtained from the electronic part [27] of the polarizability $\left(\alpha^{e}\right)$, the first $\left(\beta^{e}\right)$ and second $\left(\gamma^{e}\right)$ order hyperpolarizabilities of the unit cell as:

$$
\begin{gathered}
\epsilon_{i j}=\delta_{i j}+4 \pi \alpha_{i j}^{e} / V \\
\chi_{i j k}^{(2)}=2 \pi \beta_{i j j}^{e} / V \\
\chi_{i j k l}^{(3)}=2 \pi \gamma_{i j k l}^{e} /(3 V)
\end{gathered}
$$


where $V$ is the volume of the unit cell and $\delta_{i j}$ the Kronecker delta. Note that an ideal cubic structure features one independent $\epsilon_{i i}$ and two $\chi_{i i i i}^{(3)}$ and $\chi_{i i j j}^{(3)}$ components, respectively, while the total second order non-linear optical response $\chi^{(2)}$ vanishes.

Bearing in mind that the computation of third order susceptibilities of molecules, polymers and solids greatly depend on the quantum chemical method applied, [28-30] we computed the third order optical nonlinearities of pure and doped bulk systems using three DFT functionals within the generalized gradient approximation (GGA). These are the pure Perdew, Burke, and Ernzerhof (PBE) [31] exchange-correlation functional, its hybrid counterpart PBE0 [32] and the Becke, three-parameter, Lee-Yang-Parr exchange-correlation functional (B3LYP). [33] The first two functionals, namely PBE and PBE0, have been used in previous studies, [25,34] involving the structural and electronic properties of YSZ while the popular B3LYP, was chosen in order to check the robustness of the results. All periodic calculations have been performed at the static limit with a developer's version of CRYSTAL17 software [35] that allows analytic computations of non-linear optical (NLO) properties of infinite periodic systems. We also performed benchmark computations focusing on the molecular second dipole hyperpolarizabilities of $\left(\mathrm{ZrO}_{2}\right)_{\mathrm{n}}$ nanoclusters at $\mathrm{CCSD}(\mathrm{T})$ (coupled cluster including single, double and triple excitations) and MP2 (second order Möller-Plesset perturbation theory) levels using the GAUSSIAN09 [36] suit of programs.

Table 1. Cell parameter $a(\AA)$, bandgap $E_{g}(\mathrm{eV})$, electronic contribution of the dielectric constant $\epsilon=\epsilon_{i i}$ and third order susceptibility components $\chi_{i i i i, i i j j}^{(3)}\left(10^{-21}\right) \mathrm{m}^{2} \mathrm{~V}^{-2}$ of c- $\mathrm{ZrO}_{2}$ computed with the PBE, PBE0 and B3LYP functionals.

\begin{tabular}{ccccc}
\hline Method & PBE & PBE0 & B3LYP & Exp \\
\hline$a$ & 5.127 & 5.090 & 5.132 & $5.09[37], 5.135[38]$ \\
$E_{g}$ & 3.1 & 5.4 & 4.9 & $4.5[39], 4.6[40]$ \\
$\epsilon$ & 5.49 & 4.43 & 4.57 & $4.46^{*}$ \\
$\chi_{i i i i}^{(3)}$ & 7.60 & 1.53 & 1.91 & \\
$\chi_{i i j j}^{(3)}$ & 3.80 & 1.25 & 1.52 & \\
\hline
\end{tabular}

${ }^{*}$ Calculated from Ref. [41] at $\lambda=1500 \mathrm{~nm}$.

Third order nonlinearities of $\mathbf{c}-\mathrm{ZrO}_{2}$. The results of the respective computations are summarized in Table 1 where we present lattice constants, band gaps, the electronic contribution to the dielectric constant and the two independent $\chi^{(3)}$ tensorial components of $\mathrm{c}-\mathrm{ZrO}_{2}$. For the sake of briefness a more detailed discussion of these outcomes are given in Appendix. Here we will only mention that the observed method behavior, characterized by notable oscillations in the computed bulk-nonlinearities is in line with previous studies [28-30] performed on finite atomic semiconductor nanoclusters. Interestingly, the current computations reveal that the known pathology of pure DFT functionals effect, concerns as well the computed dielectric constants for which experimental are available (see values listed in Table 1). Nonetheless, and in-spite of the obvious deviations, the obtained results allow us to make the following deductions. First, for $\mathrm{c}-\mathrm{ZrO}{ }_{2}$, characterized by a rather wide bandgap, all three DFT methods yielded third order susceptibilities of the same order of magnitude varying between 7.6 and $1.53 \times 10^{-21} \mathrm{~m}^{2} \mathrm{~V}^{-2}$ for the tensorial component $\chi_{i i i i}^{(3)}$ and between 3.8 and $1.25 \times 10^{-21} \mathrm{~m}^{2} \mathrm{~V}^{-2}$ for $\chi_{i i j j}^{(3)}$. Second, 
considering that $\chi^{(3)}$ can be written as an infinite sum of terms [42] inversely proportional to the cubic power of transition energies, the obtained outcomes at PBE0, B3LYP and PBE levels indicate that as we approach the experimental band gap value from above/below, one should expect larger/smaller theoretical values for both $\chi_{i i i i}^{(3)}$ and $\chi_{i i j j}^{(3)}$. Third, taking into account that pure DFT functionals, as PBE, systematically overshoot the (non)linear optical properties of molecular systems [43] we expect that the upper limit of the theoretically determined components $\chi_{i i i i}^{(3)} / \chi_{i i j j}^{(3)}$ of $\mathrm{c}-\mathrm{ZrO}_{2}$ should be considerably lower than 7.6 and $3.8 \times 10^{-21} \mathrm{~m}^{2} \mathrm{~V}^{-2}$, respectively. The latter conclusions are supported by a brief DFT functional performance assessment, on the computation of the molecular second dipole hyperpolarizabilities of three $\left(\mathrm{ZrO}_{2}\right)_{\mathrm{n}}$ nanoclusters, [44] namely, $\mathrm{Zr}_{4} \mathrm{O}_{8}, \mathrm{Zr}_{8} \mathrm{O}_{16}$ and $\mathrm{Zr}_{15} \mathrm{O}_{30}$ with respect to $\mathrm{CCSD}(\mathrm{T})$ and MP2 ab-initio post Hartree-Fock methods. The respective computations, presented in Appendix, exposed in addition the pivotal importance of electron correlation effects on the third order optical nonlinearities of $\left(\mathrm{ZrO}_{2}\right)_{\mathrm{n}}$ species.

Doping and vacancy/dopant distribution effects on the optical nonlinearities of YSZ. To conduct the respective structure-property investigation, we considered doped bulk YSZ systems of two concentrations in $\mathrm{Y}_{2} \mathrm{O}_{3}$, namely, 33\% and 3.2\% mol. For the highest dopant concentration, two different compensated unit cells were built upon the face centered cubic (fcc) Fm $\overline{3} \mathrm{~m}$ cell of Zirconium dioxide (volume $=131.9 \AA^{3}, E_{g}=5.37 \mathrm{eV}$ ) by removing one $\mathrm{O}$ atom from its tetrahedral site creating an oxygen vacancy. Then, two out of the four nearest neighboring (NN) $\mathrm{Zr}$ atoms of the corresponding vacancy were substituted by an equal number of $\mathrm{Y}$ atoms, delivering two non-equivalent $\mathrm{NN}$ crystal structures of $\mathrm{Y}_{2} \mathrm{O}_{3}, 2 \mathrm{ZrO}_{2}$. The equilibrium nuclear geometry of each unit-cell and their optical nonlinearities were optimized with the PBE0 functional. In the case of $3.2 \%$ mol. in $\mathrm{Y}_{2} \mathrm{O}_{3}$, we considered the ten most stable nonequivalent configurations reported by Parkes et al. [25] the crystal structures of which were built upon a doped $2 \times 2 \times 2$ super cell of $\mathrm{c}-\mathrm{ZrO}_{2}$, optimized with the PBE functional.

Table 2. Unit cell volume $\mathrm{V}\left(\AA^{3}\right)$, band gap $E_{g}(\mathrm{eV})$, electronic contribution to the dielectric $\epsilon_{x x}=\epsilon_{y y}$ and $\epsilon_{z z}$ components, and Kerr (IDRI) effect third order susceptibility $\chi_{x x x x}^{(3)}=\chi_{y y y y}^{(3)}, \chi_{z z z z}^{(3)}$ and $\chi_{x x y y}^{(3)}, \chi_{x x z z}^{(3)}=\chi_{y y z z}^{(3)}$ components $\left(10^{-21}\right.$ $\mathrm{m}^{2} \mathrm{~V}^{-2}$ ) of two non-equivalent local crystal structures of YSZ 33\%mol. in $\mathrm{Y}_{2} \mathrm{O}_{3}$ (see Figure 2). All properties have been computed with the PBE0 functional.

\begin{tabular}{ccc}
\hline & YSZ-A & YSZ-B \\
\hline $\mathrm{V}$ & 132. & 138. \\
$E_{g}$ & 5.6 & 5.6 \\
$\epsilon_{x x}$ & 3.7 & 3.5 \\
$\epsilon_{z z}$ & 3.5 & 3.3 \\
$\chi_{x x x x}^{(3)}$ & 0.93 & 0.69 \\
$\chi_{z z z z}^{(3)}$ & 0.86 & 0.89 \\
$\chi_{x x y y}^{(3)}$ & 0.70 & 0.65 \\
$\chi_{x x z z}^{(3)}$ & 0.62 & 0.62 \\
\hline
\end{tabular}

Computed electronic and optical properties of the two crystal configurations of YSZ 33\%mol. in $\mathrm{Y}_{2} \mathrm{O}_{3}$ are given in Table 2. Both crystal structures are characterized by equal bandgap values and reduced third order nonlinearities to those obtained for pure $\mathrm{c}-\mathrm{ZrO}_{2}$ (see Table 1). Judging 


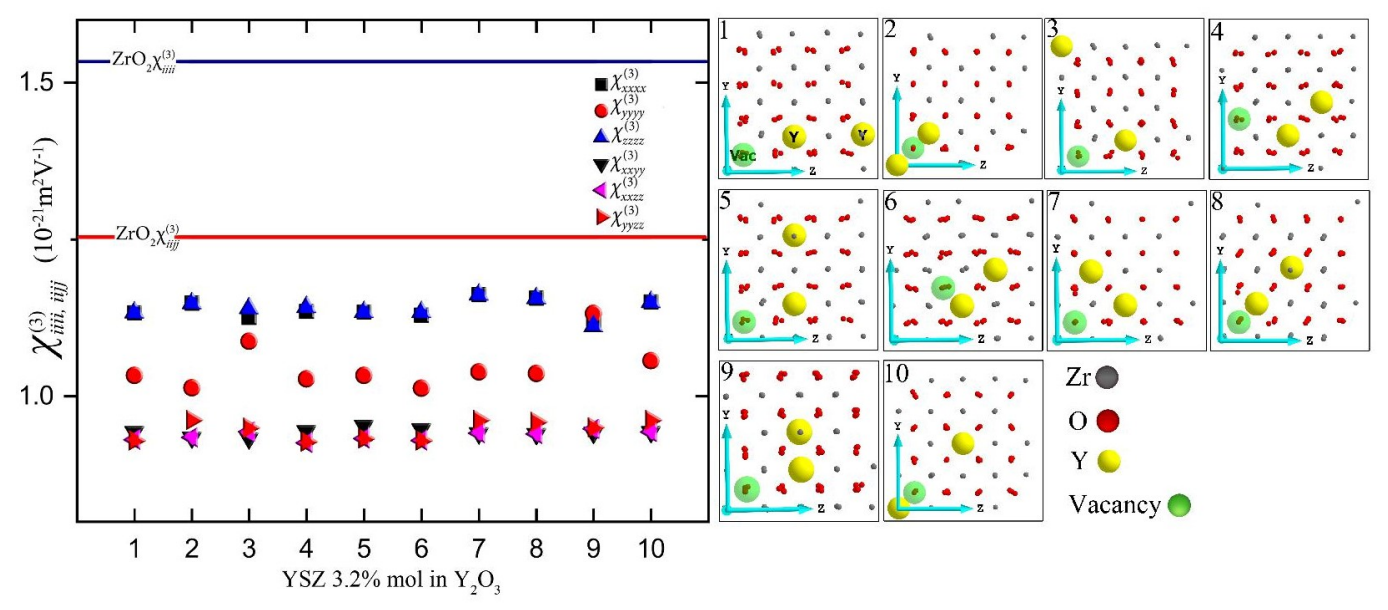

Fig. 1. Variation of the third order susceptibility tensorial components $\left(\chi_{i i i i, i i j j}\right)$ of YSZ 3.2\% mol. in $\mathrm{Y}_{2} \mathrm{O}_{3}$ as a function of the relative position between $\mathrm{Y}$ dopants (yellow spheres) and $\mathrm{O}$-vacancies (green spheres). The unit cells [25] of each configuration considered, representing symmetry in-equivalent vacancy/dopant distributions, are schematically given at the right. Solid lines represent the corresponding susceptibility components of $\mathrm{c}-\mathrm{ZrO}_{2}$. All values have been computed at the PBE0 level of theory.

from the relative strengths of each component along the $x \equiv y$ and $z$ axis we see that although the creation of an $\mathrm{O}$ vacancy and the replacement of two $\mathrm{Zr}$ atoms with $\mathrm{Y}$ reduces the cubic symmetry of the host, the third order NLO responses remain quasi-isotropic in character since $\chi_{x x x x, x x y y}^{(3)} \approx \chi_{z z z z, x x z z}^{(3)}$. Hence, even for a relatively high Yttria concentration, YSZ inherits most of the isotropic nonlinear optical nature of $\mathrm{c}-\mathrm{ZrO}_{2}$. A similar property trend is obtained for all configurations of $\mathrm{Y}_{2} \mathrm{O}_{3} 3.2 \% \mathrm{~mol}$. considered here.

As seen in Figure 1, the influence of the vacancy/dopant distribution on the properties of interest is practically negligible. Furthermore, for both concentrations we also computed the corresponding second order susceptibilities. In all cases, the computed second harmonic generation $\chi^{(2)}(-2 \omega ; \omega, \omega)$ for $\omega=0.8 \mathrm{eV}$ (corresponding to the wavelength $\lambda=1550 \mathrm{~nm}$ ) have proven considerably small. Indeed, the largest absolute xyz-component value of $\chi^{(2)}$ for the most stable YSZ 33\%mol. structure is lower than $0.014 \mathrm{pmV}^{-1}$.

In comparison to the $\mathrm{c}-\mathrm{ZrO}_{2}$, the weak band gap opening of about $0.2 \mathrm{eV}$ observed in YSZ $33 \%$ mol. in $\mathrm{Y}_{2} \mathrm{O}_{3}$ should not justify the observed lowering in the third order susceptibilities. Hence, bearing in mind that the third order nonlinearities of charge transfer insulators [45] should depend on the amount of electron and hole states lying near the gap, the observed effect could be primarily attributed to the doping induced reduction of the density of low lying hole-states in the conduction band. This is very well illustrated by the projected density of states (PDOS) of YSZ $33 \%$ mol. (see Figure $2 b$ ) where the DOS projected on $\mathrm{Zr}$ atoms (3p in character), located at the bottom of the conduction band, drastically decreases after the replacement of two $\mathrm{Zr}$ atoms with an equal number of $\mathrm{Y}$. On the other hand, the creation of an $\mathrm{O}$ vacancy does not bring important changes in the valence band (O-2p-in-character) of $\mathrm{c}-\mathrm{ZrO}_{2}$. These are two enlightening trends suggesting that the third order nonlinear optical responses of doped $\mathrm{c}-\mathrm{ZrO}_{2}$ could be further improved with doping agents that are able to deliver a significant increase of low lying hole-states.

Concentration dependence. So far, the obtained computational data revealed that the cubic phase of pure $\mathrm{c}-\mathrm{ZrO}_{2}$ is characterized by third order nonlinearities lying above the limit of 1. $\times 10^{-21} \mathrm{~m}^{2} \mathrm{~V}^{-2}$. Also, computations on two different concentrations of YSZ (33 and 3.2\% 
(a)

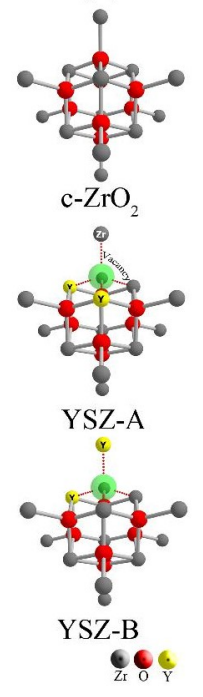

(b)
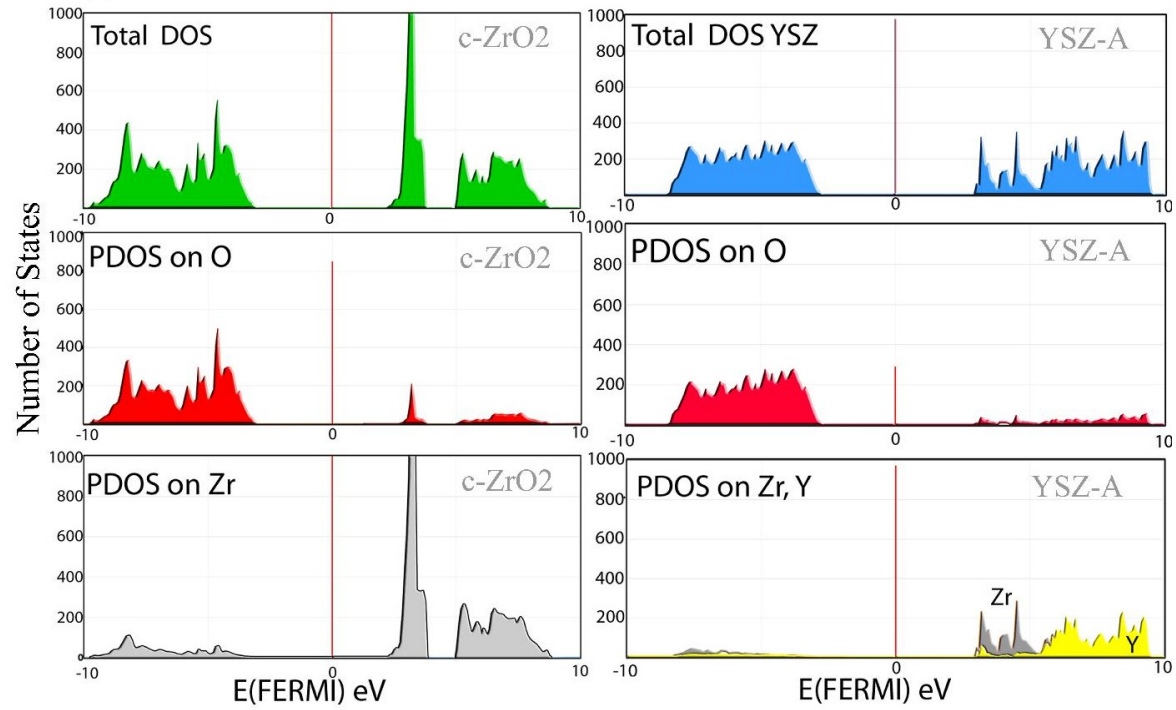

Fig. 2. (a) Symmetry non-equivalent local crystal structures of YSZ 33\%mol. in $\mathrm{Y}_{2} \mathrm{O}_{3}$. Zirconium, Oxygen and Yttrium atoms are in grey, red and yellow, respectively, and the vacancy is in green. See Data File 1 and Data file 2 for the unit-cell fractional coordinates of YSZ-A and YSZ-B, respectively. (b) Total and projected density of states of $\mathrm{c}-\mathrm{ZrO}_{2}$ and $\mathrm{YSZ}-\mathrm{A}$.

in $\mathrm{Y}_{2} \mathrm{O}_{3}$ ) provided solid proofs that $\mathrm{Y}$-doping delivers a notable lowering of the respective nonlinear optical responses whatever the vacancy/dopant distribution in the crystalline phase. In this section we briefly focus on variation of the $\chi_{i i i i}^{(3)}$, and $\chi_{i i j j}^{(3)}$ components of YSZ as a function of the $\mathrm{Y}_{2} \mathrm{O}_{3}$ concentration. For this task we considered three concentrations of YSZ, namely $7 \%$ mol., $14 \%$ mol. and $33 \%$ mol. All unit cells of the doped bulk systems were built upon compensated super-cells of c- $\mathrm{ZrO}_{2}$ of appropriate sizes in order to obtain each of the desired concentration in $\mathrm{Y}_{2} \mathrm{O}_{3}$. Unit-cell fractional coordinates of the four $\mathrm{YSZ}$ systems considered, 7\%, $14 \%, 33 \%-\mathrm{A}$ and $33 \%-\mathrm{B}$ are reported in the supplementary material.

In every local crystal structure, NN doping method has been adopted without performing further structural investigations for the determination of the most stable atomistic crystal structure. The obtained property evolution, depicted in Figure 3, clearly demonstrates that YSZ third order susceptibilities increase in a rather monotonic fashion while decreasing the concentration in $\mathrm{Y}_{2} \mathrm{O}_{3}$. Therefore, the optimal YSZ bulk system for photonic applications, built upon c- $\mathrm{ZrO}_{2}$, should feature the lowest concentration in $\mathrm{Y}_{2} \mathrm{O}_{3}$ that ensures the stability of the cubic phase of the respective crystal at room temperature.

\section{Experimental characterization}

The Intensity Dependent Refractive Index (IDRI) Kerr property of YSZ has been characterized via the Kerr-induced Self Phase Modulation (SPM), in a YSZ-based waveguide directly fabricated in the YSZ $8 \%$ mol. thin film.

The growth of high quality YSZ thin film is performed with an optimized Pulsed-Laser Deposition (PLD) process on sapphire (0001), described in details reference. [22] We demonstrated that the [001] growth direction of YSZ can be largely promoted with up to six in-plane grains orientations separated by 15 degrees. The characterization by X-Ray Diffraction (XRD) of the $\mathrm{H}=300 \mathrm{~nm}$ 


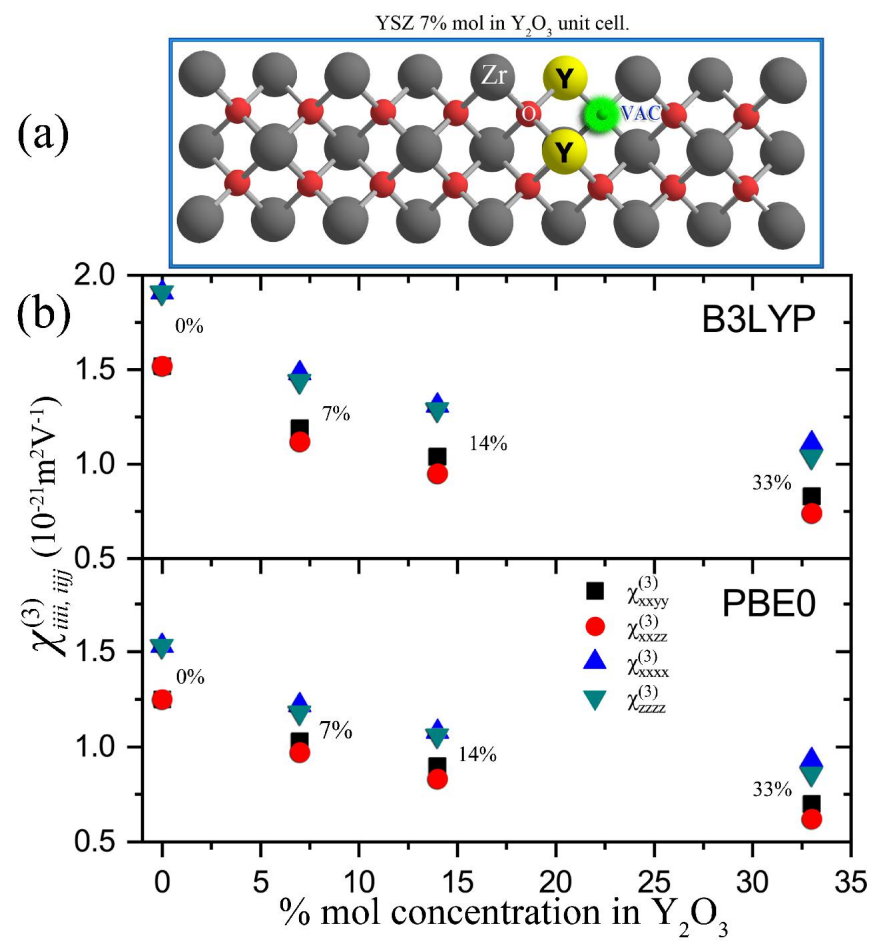

Fig. 3. (a) Unit cell of YSZ 7\%mol. considered in this study. (b) Evolution of $\chi_{i i i i}^{(3)}$ and $\chi_{i i j j}^{(3)}$ of YSZ as a function of the concentration in $\mathrm{Y}_{2} \mathrm{O}_{3}$ computed with the B3LYP and PBE0 functionals and the smallest ECP basis set used in this work. All local crystal structures have been optimized at the PBE0 level of theory, see Data File 3 and Data file 4 for the unit-cell fractional coordinates of YSZ 7\% mol and YSZ 14\%mol, respectively.

thick film used in this work is presented Figure $4 \mathrm{~d}$ and $4 \mathrm{e}$. The $2 \theta-\omega$ XRD scan mode confirms the only [001] YSZ growth direction on sapphire whereas the mosaicity is estimated via the Full Width at Half Maximum (FWHM $=0.03^{\circ}$ ) of the (002) YSZ peak of the $\omega$-scan.

YSZ waveguides are designed to confine and propagate a single quasi-TE optical mode in telecom wavelength range. After an optimized fabrication process based on electron-beam lithography and ion-beam etching techniques, waveguide dimensions are measured by Atomic-Force Microscopy (AFM), as represented with the topographic profile Figure $4 \mathrm{~b}$. The etching depth is $\mathrm{D}=80 \mathrm{~nm}$ and waveguides width $\mathrm{W}$ about $760 \mathrm{~nm}$. Propagation losses $\alpha=3.2 \mathrm{dBcm}^{-1}$ at $\lambda=1550$ $\mathrm{nm}$ are measured for the quasi-TE mode thanks to the comparison of transmission levels from different waveguide on the sample, long from $\mathrm{L}=0.8 \mathrm{~mm}$ to $\mathrm{L}=5.8 \mathrm{~mm}$. This loss measurements are performed with grating couplers, located at each waveguide extremity and fabricated with the same process in the YSZ thin film.

As a nonlinear phenomenon, the Kerr effect characterization in YSZ waveguides strongly depends on the light-matter interaction and thus on the injected optical power. In order to improve the light injection we have therefore considered the butt coupling configuration and the longest 
(a)

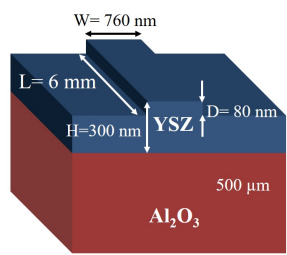

(d)

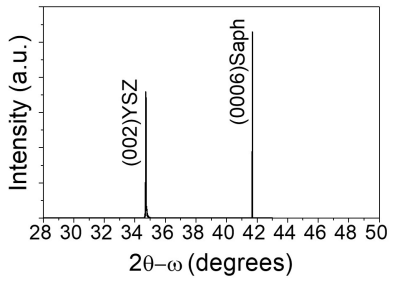

(b)

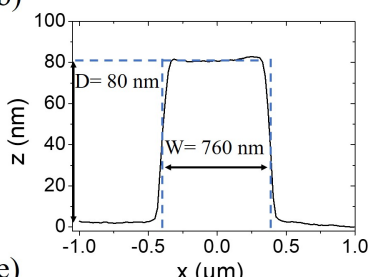

(e)

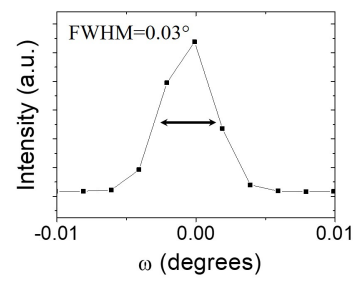

(c)

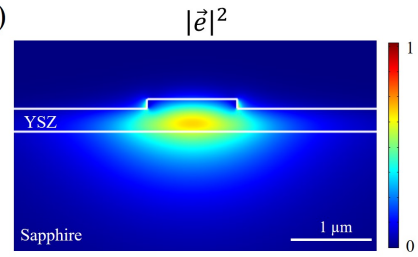

(f)

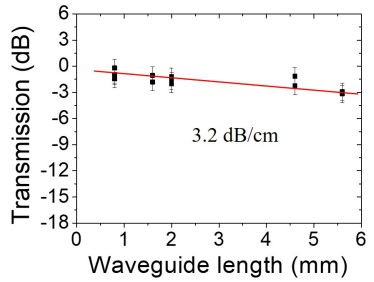

Fig. 4. (a) Schematic view of a YSZ-based rib waveguide, designed and fabricated for single-mode quasi-TE propagation in the $\mathrm{H}=300 \mathrm{~nm}$ YSZ thin film. (b) YSZ waveguide geometry is characterized by Atomic-Force Microscopy (AFM). Dimensions of the waveguide are $D=80 \mathrm{~nm}$ for the etching depth and $\mathrm{W}=760 \mathrm{~nm}$ for the width. (c) Simulation of fundamental TE mode under the experimental geometrical values of the YSZ waveguide. (d) X-Ray Diffraction (XRD) of the YSZ thin film studied on sapphire. The 2 $\theta-\omega$ XRD scan presents both (002) and (0006) diffraction peak from YSZ and sapphire substrate respectively, confirming the only one [001] YSZ growth direction. (e) The $\omega$-scan reveals the mosaïcity of the (001) YSZ plans with a Full-Width at Half Maximum FWHM=0.03 . (f) Propagation losses $\alpha=3.2 \mathrm{dBcm}^{-1}$ are estimated at $\lambda=1550 \mathrm{~nm}$ thanks to the transmission level of different waveguides, long from 0.8 to $5.8 \mathrm{~mm}$.

waveguide available on the chip with a length of $\mathrm{L}=5.8 \mathrm{~mm}$.

The experimental set-up used for the characterization of the YSZ samples is described in referenc. [46] It consists of a mode locked Erbium doped fiber laser that delivers 150 fs duration pulses with a repetition rate of $\mathrm{F}=50 \mathrm{MHz}$ for a wavelength of $\lambda=1580 \mathrm{~nm}$. After passing through a polarization beam splitter that guarantees the polarization state to excite the fundamental TE mode, the pulses are shaped through a grating based stretcher that fixes the pulse spectrum following a quasi-rectangular shape of variable widths and introduces an adjustable dispersion coefficient $\phi^{(2)}$. In the Fourier limit, the autocorrelation pulse duration has been measured equals to $T_{0}=2 \mathrm{ps}$. The polarized beam that exits the stretcher is injected via butt-coupling, using a microscope objective (X20), to the YSZ waveguide which matches the excitation guided mode in the taper. After the sample, another polarization beam splitter is used to verify the transmitted polarization state.

We first probed thanks to this set-up the nonlinear absorption of YSZ by measuring the output light power $P_{\text {out }}$ for different injected powers $P_{\text {in }}$ in the YSZ waveguide. The result plotted in Figure 5a shows a linear behavior, indicating the absence of Two-Photon Absorption (TPA) as it was expected due to the YSZ bandgap energy around $\mathrm{Eg}=4.5 \mathrm{eV}$. [39,40] From the slope, and considering the two facets of the waveguide equivalent, we also extracted the transmission coefficient of about $\kappa_{F}=9.1 \%$. The comparison of the output spectra for different input power $P_{i n}$, Figure $5 \mathrm{~b}$, reveals a spectral broadening for higher power due to the Kerr effect induced by the constituting material of the waveguide.

In absence of TPA, the nonlinear effective susceptibility of the waveguide can be written as $\operatorname{Re}\left[\gamma_{w g}\right]=\phi_{N L} /\left(\kappa_{F} P_{i n} L_{e f f} \eta\right)$, with $\phi_{N L}$ the nonlinear phase shift and $L_{\text {eff }}$ the effective 
(a)

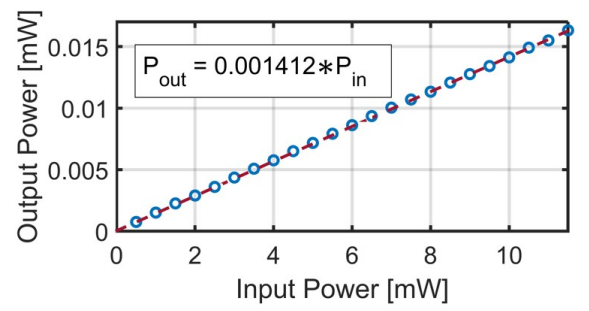

(c)

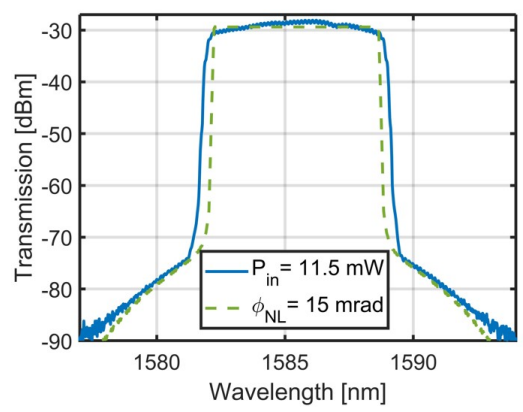

(b)

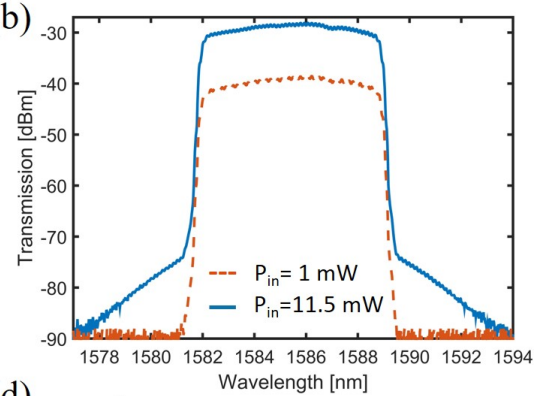

(d)

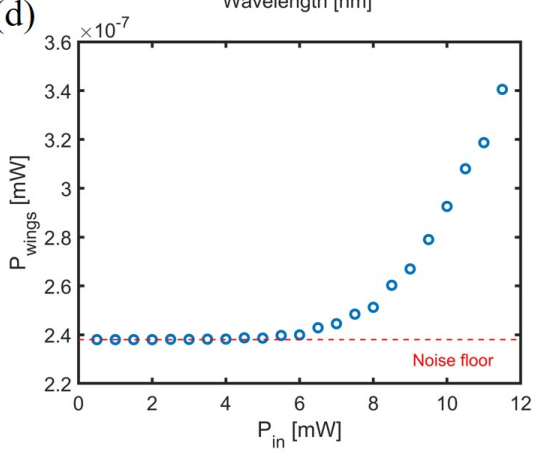

Fig. 5. (a) $P_{\text {out }}$ vs. $P_{\text {in }}$ curve at $\lambda=1580 \mathrm{~nm}$ revealing the absence of Two-Photon Absorption (TPA), in agreement with the bandgap energy of $E g>5 \mathrm{eV}$. (b) Optical transmission at low (dashed orange) and high (solid blue) input powers. (c) Simulation (dashed red) of the spectrum transmits through a YSZ-based waveguide calculated with the experimental parameters. (d) Experimental measurement of the power in the generated frequencies.

length given by $L_{\mathrm{eff}}=(1-\exp (-\alpha L)) / \alpha$ where $\alpha$ is the linear propagation losses, and $\eta=$ $1 /\left(F \int_{0}^{1 / F}|U(t)|^{2} d t\right)$ with $U(t)$ the normalized temporal shape of the pulse.

The mode simulation Figure $4 \mathrm{c}$ reveals the distribution of the electric field intensity propagating through the YSZ waveguide. Being the substrate for the growth of the YSZ thin film, sapphire also interacts with a part of the input power. Then, to extract the nonlinearities of YSZ, both materials have to be considered in the nonlinear response. In a first approach, we can rewrite the waveguide effective nonlinear susceptibility as the contribution of the two nonlinear materials such as

$$
\operatorname{Re}\left[\gamma_{w g}\right]=\frac{2 \pi}{\lambda_{0} A_{N L}}\left(\Gamma_{\mathrm{YSZ}} n_{2}^{\mathrm{YSZ}}+\Gamma_{\mathrm{Al}_{2} \mathrm{O}_{3}} n_{2}^{\mathrm{Al}_{2} \mathrm{O}_{3}}\right)
$$

where $\Gamma_{Y S Z}=36.9 \%$ and $\Gamma_{A_{2} O_{3}}=63.0 \%$ are the overlapped power confined in the two materials, $\lambda_{0}$ the wavelength of the pump, $A_{N L}$ the nonlinear area calculated from the mode simulation and $n_{2}$ the nonlinear refractive index. The nonlinear Kerr index from sapphire was taken from reference [47] and equals to $n_{2}^{\mathrm{Al}_{2} \mathrm{O}_{3}} \approx 3 \times 10^{-20} \mathrm{~m}^{2} \mathrm{~W}^{-1}$. For the maximum input average power $P_{i n}=11.5 \mathrm{~mW}$, the corresponding nonlinear phase shift $\phi_{N L}$ is simulated to be $15 \mathrm{mrad}$ as represented in Figure 5c. This value leads to an effective Kerr nonlinear coefficient in YSZ equals to $n_{2}^{Y S Z} \approx 4.0 \pm 2 \times 10^{-19} \mathrm{~m}^{2} \mathrm{~W}^{-1}$, being one order of magnitude larger than in sapphire and similar to the silicon nitride. [48] Furthermore, we have performed the analysis of the spectral broadening behavior by plotting in Figure $5 \mathrm{~d}$ the power contained in the symmetric wings $P_{\text {wings }}$ of the measured output spectra for the different input powers. For input power lower than $6 \mathrm{~mW}, P_{\text {wings }}$ is constant, limited to the noise floor of the optical spectrum analyser. For 
higher powers, a quadratic variation of $P_{\text {wings }}$ is observed in accordance with the variation of the Kerr induced spectral broadening which is expected to be proportional to $P_{i n}^{2}$ for $\phi_{N L} \ll 1$. [49]

\section{Discussion}

As YSZ is a crystal, $n_{2}^{Y S Z}$ is a tensor as well as the third order nonlinear susceptibility $\chi_{Y S Z}^{(3)}$. The in-plane orientation of YSZ grains in the waveguide is not well defined like in classical silicon substrate and then the electric-field direction of the pump is not aligned along only one crystallographic direction. Thanks to a complete material characterization of our films, [22] we considered as a first approximation that the electric field of the pump is aligned equiprobably in the [100] and [110] directions. In this case, the experimental value $n_{2}^{Y S Z}$ becomes an effective value $\widehat{n}_{2}^{Y S Z}$ which can be compared to the theory via an effective third order nonlinear susceptibility $\hat{\chi}_{Y S Z}^{(3)}$, defined as follow

$$
\widehat{\chi}_{Y S Z}^{(3)}=\frac{\chi_{Y S Z,<100>}^{(3)}+\chi_{Y S Z,<110>}^{(3)}}{2}
$$

where it has been demonstrated that for a cubic crystal

$$
\chi_{Y S Z,<100>}^{(3)}=\chi_{Y S Z, x x x x}^{(3)}
$$

and

$$
\chi_{Y S Z,<110>}^{(3)}=\frac{\chi_{Y S Z, x x x x}^{(3)}+3 \chi_{Y S Z, x x y y}^{(3)}}{2}
$$

Using the calculated values for YSZ 7\% mol., presented in Table 3, we found a theoretical effective susceptibility of $\widehat{\chi}_{Y S Z}^{(3)}=2.00 \times 10^{-21} \mathrm{~m}^{2} \mathrm{~V}^{-2}$ at B3LYP level of theory, leading to the birefringence $\Delta n=\widehat{n}_{2} \mathrm{I}$, where I is the laser radiation power per unit surface, with the in-plane refractive index: $\widehat{n}_{2}=3 \widehat{\chi}^{(3)} / 4 c \varepsilon_{0} \epsilon=1.3 \times 10^{-19} \mathrm{~m}^{2} \mathrm{~W}^{-1}(\epsilon$ is the relative dielectric in-plane $\epsilon_{x x}$-component, $\varepsilon_{0}$ is the electric permittivity of vacuum and $c$ is the light celerity).

Table 3. Electronic contribution to the dielectric susceptibilities $\left(\epsilon_{x x}\right)$, third order susceptibilities $\left(\chi_{x x x x, x x y y}^{(3)} \times 10^{-21} \mathrm{~m}^{2} \mathrm{~V}^{-2}\right)$, effective third order susceptibilities $\left(\widehat{\chi}^{(3)} \times 10^{-21} \mathrm{~m}^{2} \mathrm{~V}^{-2}\right)$ and nonlinear refractive index $\left(\widehat{n}_{2} \times 10^{-19} \mathrm{~m}^{2} \mathrm{~W}^{-1}\right)$ of YSZ $7 \%$ mol. in $\mathrm{Y}_{2} \mathrm{O}_{3}$ (Figure 3a) computed at the PBE0 and B3LYP levels of theory on PBE0 optimized local crystal structures. For $\mathrm{Zr}, \mathrm{Y}$ atoms we used the pseudopotential (ECP) basis set of Hay and Wadt (Zr[3s1p1d], Y[2s1p1d]), for O atoms the Durand-Barthelat ECP basis ([2s1p])

\begin{tabular}{cccccc}
\hline & & & & & \\
& $\epsilon_{x x}$ & $\chi_{x x x x}^{(3)}$ & $\chi_{x x y y}^{(3)}$ & $\widehat{\chi}^{(3)}$ & $\widehat{n}_{2}$ \\
PBE0 & 4.24 & 1.20 & 0.99 & 1.64 & 1.1 \\
B3LYP & 4.33 & 1.48 & 1.19 & 2.00 & 1.3 \\
B3LYP(+spd) & 4.75 & 1.65 & 1.23 & 2.16 & 1.3 \\
& & & & & \\
\hline
\end{tabular}

The theoretical value for YSZ 7\%mol. is very close to the one of pure c- $\mathrm{ZrO}_{2}$ which at the same level of theory is estimated to lie only 1.2 times higher (e.g. $1.3 \times 10^{-19} \mathrm{~m}^{2} \mathrm{~W}^{-1}$ for YSZ vs $1.6 \times 10^{-19} \mathrm{~m}^{2} \mathrm{~W}^{-1}$ for $\mathrm{c}-\mathrm{ZrO}_{2}$ at B3LYP level of theory). 
The convergence between experiment and theory is expected to improve if two additional factors are to be taken into account. The first concerns the vibrational contributions to the third order NLO responses of the systems considered. To address these effects we computed Raman intensities with the CRYSTAL code, which depend on the variation of the dipole polarizability of the unit cell with respect to atomic displacements. The outcomes of this brief investigation at the PBE0 level of theory and the basis set of Table 3 suggest that the total IDRI second unit-cell hyperpolarizabilities [50] $\left(\gamma_{\mathrm{IDRI}}^{\mathrm{tot}}\right)$ of the pure and doped $\mathrm{c}-\mathrm{ZrO}_{2}$, defined as $\gamma_{\mathrm{IDRI}}^{\mathrm{tot}}=\gamma_{\mathrm{IDRI}}^{\mathrm{vib}}+\gamma^{e}$, should be about $10 \%$ larger than $\gamma^{e}$, regardless the laser "high-frequency" $\left(\sim 6500 \mathrm{~cm}^{-1}\right)$. The second factor precisely, should involve frequency dispersion effects on $\gamma^{e}$ which have not been treated here under periodic boundary conditions due to software limitations. Nevertheless, we estimate that for the experimental laser wavelength, corresponding to a photon energy of $0.8 \mathrm{eV}$ $(\lambda=1550 \mathrm{~nm})$, and a solid with a gap around $5 \mathrm{eV}$, the electronic part of IDRI- $\chi^{(3)}(-\omega ; \omega,-\omega, \omega)$ should experience a maximum increase of $10 \%$, under the hypothesis that the UV-absorption spectrum has a strong absorption peak precisely at the gap. The respective guesstimate is further supported by time dependent DFT (TD-DFT) sum-over-state (SOS) computations conducted on two out of the three nanoclusters considered in this work, namely, $\left(\mathrm{ZrO}_{2}\right)_{4}$ and $\left(\mathrm{ZrO}_{2}\right)_{8}$ (for more details see Appendix). Furthermore, owed to its positive coefficient of thermal expansion, the cell parameter of $\mathrm{ZrO}_{2}$ is expected to increase as the temperature rises. This effect, not taken into account in the calculation, could deliver an additional small increase of $\chi^{(3)}$. Similarly, the strain induced in the YSZ thin film by the sapphire substrate during the growth could be also considered as an additional source of the observed deviation between theory and experiment.

\section{Conclusion}

In conclusion, we present in this work a detailed theoretical and experimental study of the third order nonlinearities of YSZ. The IDRI Kerr property has been first predicted with ab-initio calculations, for different unit cell configurations and compositions. As the nonlinearities increase when the Yttrium-doping concentration decreases, we have experimentally fabricated and characterized YSZ waveguides with a minimum of $8 \% \mathrm{~mol}$. of $\mathrm{Y}_{2} \mathrm{O}_{3}$, ensuring the cubic structure. The nonlinearity birefringence $n_{2}^{Y S Z} \approx 4.0 \pm 2 \times 10^{-19} \mathrm{~m}^{2} \mathrm{~W}^{-1}$ experimentally measured is compared to the predicted static theoretical value: $\widehat{n}_{2} \approx 1.3 \times 10^{-19} \mathrm{~m}^{2} \mathrm{~W}^{-1}$ which is expected to rise up to $\widehat{n}_{2} \approx 1.6 \times 10^{-19} \mathrm{~m}^{2} \mathrm{~W}^{-1}$ if vibrational and dispersion effects are to be taken into account.

The obvious convergence between experiment and theory demonstrates the robustness of our method to characterize the nonlinearities of new materials. Furthermore, the computational results exposed that for low concentrations in $\mathrm{Y}_{2} \mathrm{O}_{3}$, YSZ IDRI Kerr should lie very close to that of pure $\mathrm{c}-\mathrm{ZrO}_{2}$ which is not stable in ambient conditions. The latter outcome originates from the inverse proportionality of both $\epsilon$ and $\chi^{(3)}$ to the amount of $\mathrm{Y}_{2} \mathrm{O}_{3}$ units comprised in the local atomistic structure of YSZ $\left(n_{2} \propto \chi^{(3)} / \epsilon\right)$. Hence, although Y doping weakens the third order nonlinearities of pure $\mathrm{c}-\mathrm{ZrO}_{2}$, it does not have a dramatic impact on its Kerr index (IDRI) which is of critical importance in photonic applications. Therefore, the current theoretical and experimental investigation highlights the potential of YSZ to be used as a new material for nonlinear optics.

\section{Appendix}

\section{Sample fabrication}

Sapphire (0001) substrates are cleaned with ethanol and annealed at 1200Âr̆C in air for 4 hours before deposition. Laser ablation of $8 \%$ mol. yttria-stabilized zirconia rotating ceramic target is carried out in a high vacuum stainless-steel chamber with a base pressure of $10^{-6}$ torr. The $\mathrm{KrF}$ excimer pulsed-laser $(\lambda=248 \mathrm{~nm}, 3 \mathrm{Jcm}-2,5 \mathrm{~Hz}$ repetition-rate $)$ irradiates 
a)

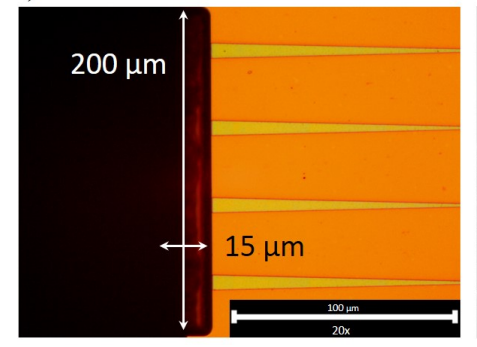

b)

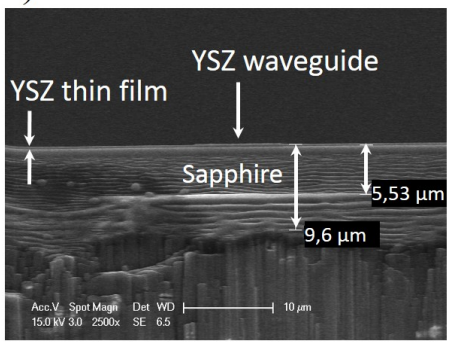

Fig. 6. Waveguides facets obtained with the two steps procedure of dicing, including classical dicing technique and Focus Ion-Beam (FIB) etching. a) Top-view of the sample edges with optical microscopy and b) cross-section observation of a waveguide facet by SEM. One of the two sides of the sample is presented from a top view figure $\mathrm{S} 2 \mathrm{a}$ ) and from a cross section figure S2 b). Whereas the first classical technique allows to mechanically dice the whole sample, the FIB technique etchs a small area, here the extremities of the waveguides, leaving highly transmissive facets.

the target with an incidence angle of $45 \hat{A} \check{r}$. The deposition occurs in a controlled oxygen atmosphere of 30 mtorr with the substrate placed at $50 \mathrm{~mm}$ from the target and heated to $800 \hat{A}$ řC. YSZ thin films on sapphire are then cooled down to room temperature under 300 torr of oxygen at $10 \hat{\mathrm{A}} \breve{\mathrm{C}} \mathrm{Cmin}^{-1}$ after deposition. Films thickness are measured with spectroscopic ellipsometry (J.A. Woollam). Structural properties and crystallinity of the film are investigated with high-resolution X-Ray Diffraction (XRD) using PANalytical X'Pert Pro diffractometer and Transmission Electron Microscopy (TEM) using an aberration probe-corrected FEI Titan Themis microscope. Roughness of the film and geometry parameters (etching depth, width...) of the rib waveguides are characterized with Atomic Force Microscopy (AFM). YSZ rib waveguides are fabricated by electron-beam lithography in a $200 \mathrm{~nm}$ thick hydrogen silsesquioxane (HSQ) electron resist layer. Charging effect during the writing, due to the insulator behavior of YSZ and sapphire, is avoided by covering the resist with a $20 \mathrm{~nm}$ thick gold layer. The etching is performed with an optimized Ion Beam Etching (IBE) technique using argon ions, accelerated with a voltage of Vaccel $=250 \mathrm{~V}$ and sputtering the sample with an incidence angle of 20Âr from the normal. Butt-coupling injection, required for third order nonlinear optical characterization, is achieved by opening each facet of the YSZ waveguides by a two steps procedure of dicing. The procedure includes firstly a mechanical dicing technique and secondly a Focus Ion-Beam (FIB) etching technique, using a FEI Scios dual beam.

\section{Computational Details}

The truncation criteria of the Coulomb and exchange infinite lattice series in the self-consistent field (SCF) calculation are controlled by five thresholds, $T_{i}$, which have been set to: $8\left(T_{1}-T_{4}\right)$ and $16\left(\mathrm{~T}_{5}\right)$. The number of k-points in the irreducible Brillouin zone (IBZ) of the reciprocal space has been fixed by the shrinking factors equal to 8 ( $35 \mathrm{k}$-points in the IBZ for the symmetric $\mathrm{c}-\mathrm{ZrO}_{2}$ structure). The default numerical integration grid (XLGRID) has been used for DFT and convergence thresholds on self-consistent-field (SCF) energy has been set to $10^{-7}$ hartree for structural optimizations (see the Crystal's manual for more details on the parameters [51]). Calculation of the electronic contribution to polarizability $\left(\alpha^{e}\right)$ and hyperpolarizabilities $\left(\beta^{e}\right.$ and $\gamma^{e}$ ) of a unit cell is done with the coupled-perturbed Hartree-Fock and Kohn-Sham (CPHF/KS) method [52] implemented in the CRYSTAL code for both finite molecular and infinite periodic 

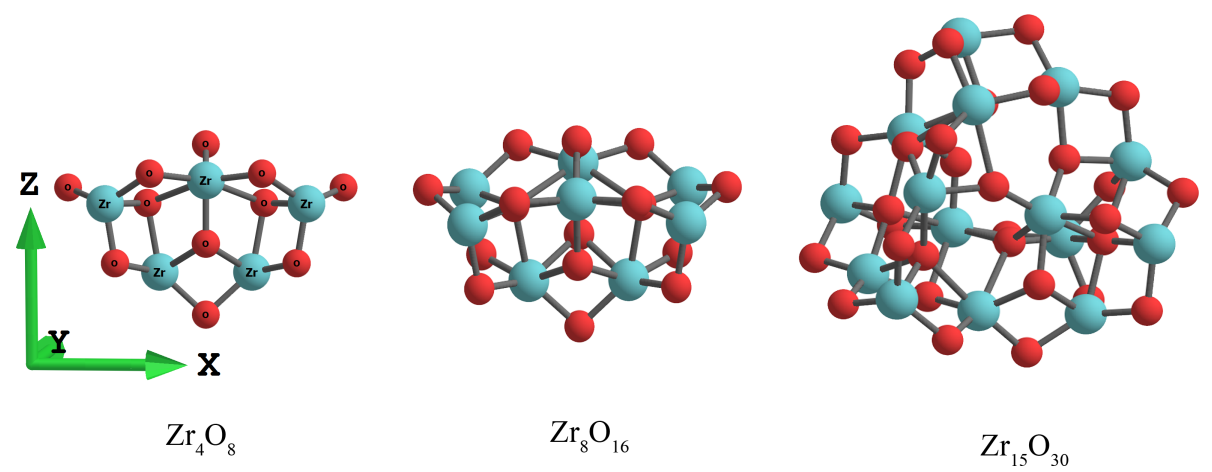

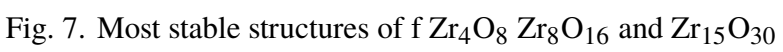

systems [53-56]. For Zr, and Y atoms we relied on pseudo-potential (ECP) basis set substrates constructed by Hay and Wadt. In this set the 12 and 11 valence electrons of $\mathrm{Zr}$ and $\mathrm{Y}$, respectively, are described by the $(5 \mathrm{sp}, 4 \mathrm{~d}) /[3 \mathrm{sp}, 2 \mathrm{~d}]$ and $(4 \mathrm{sp}, 2 \mathrm{~d}) /[2 \mathrm{sp} 1 \mathrm{~d}]$ sets of gaussian type functions, while the remaining 28 core electrons are approximated by a non-relativistic potential. For $\mathrm{O}$ atoms the Durand-Barthelat ECP basis set substrate was chosen in which the $1 s^{2}$ core electrons are approximated by a non-relativistic potential and the valence electrons are described by a (4sp)/[2s1p] all electron set. The respective basis sets have been successfully used by Ricca et al. [34] in the study of YSZ surfaces.

\section{Third order nonlinearities of $\mathrm{c}-\mathrm{ZrO}_{2}$.}

As seen in Table I of the main article, all methods considered provide cell parameters close to the experiment. In the case of the computed bandgaps, PBE0 and B3LYP hybrid functionals overestimate by $0.8(0.9)$ and $0.3(0.4) \mathrm{eV}$, respectively, the experimentally determined values reported by McComb [40] and Elizalde et al. [39]. The latter DFT methods provide also (optical) dielectric constants which are found in good agreement with available experimental data. On the other hand, the PBE pure functional delivered a band gap value which lies about $1.5 \mathrm{eV}$ lower than the experimentally determined one and overestimates the experimental dielectric constant. To obtain a rough estimation about the influence of the dynamic electron correlation on the computed bulk-nonlinearities we performed computations using the Hartree-Fock (HF) approximation which does not treat dynamic electron correlation effects. The computed HF large bandgap of $13.2 \mathrm{eV}$ and the considerably weaker HF $\chi_{i i i i}^{(3)}$ and $\chi_{i i j j}^{(3)}$ values of 0.13 and $0.19 \times 10^{-21} \mathrm{~m}^{2} / V^{2}$ respectively, demonstrate that electron correlation effects, by definition taken into account in DFT, should be of decisive importance for the accurate theoretical determination of the nonlinearities of pure $\mathrm{c}-\mathrm{ZrO}_{2}$. To check the influence of the basis set applied, we added to the initial basis set, one diffuse sp-gaussian type function for $\mathrm{Zr}$ and one d-type polarization function on each $\mathrm{O}$ atom of $\mathrm{c}-\mathrm{ZrO}_{2}$. The obtained values of $2.0 \times 10^{-21} \mathrm{~m}^{2} / \mathrm{V}^{2}$ for $\chi_{i i i i}^{(3)}$ and $1.5 \times 10^{-21} \mathrm{~m}^{2} / \mathrm{V}^{2}$ for $\chi_{i i j j}^{(3)}$, at B3LYP level, reveal that if a richer basis set is chosen, no dramatic changes on the computed bulk optical nonlinearities should be expected. Here it is important to note that the obvious choice to add a set of diffuse sp function on $\mathrm{O}$ atoms has proven impractical due to severe convergence problems in the SCF procedure. Finally, the replacement of ECP basis set for $\mathrm{O}$ by an all-electron basis (8s6sp2d)/[1s3sp2d]) [57]) did not bring important changes on the computed third order optical nonlinearities. For instance, at the PBE0 level the latter basis set yielded a value of $1.65 \times 10^{-21} \mathrm{~m}^{2} / V^{2}$ for $\chi_{i i i i}^{(3)}$ while for $\chi_{i i j j}^{(3)}$ a value of $1.25 \times 10^{-21} \mathrm{~m}^{2} / \mathrm{V}^{2}$ was obtained. 
Table 4. Electronic contribution to the second order dipole hyperpolarizability axial tensorial components of $\mathrm{Zr}_{4} \mathrm{O}_{8} \mathrm{Zr}_{4} \mathrm{O}_{8}, \mathrm{Zr}_{4} \mathrm{O}_{8}$ computed at the HF, MP2 CCSD(T) B3LYP, PBE0 and PBE levels of theory with the Def2-SVPD basis set (Zr:ECP(28 core electrons)(7s7p5d1f)/[5s4p2d1f](valence), O:(8s5p2d)/[4s3p2d]). All values have been divided by $10^{3}$ and they are given in atomic units.

\begin{tabular}{cccc}
\hline Method & $\gamma_{x x x x}$ & $\gamma_{y y y y}$ & $\gamma_{z z z z}$ \\
\hline $\mathrm{Zr}_{4} \mathrm{O}_{8}$ & & & \\
$\mathrm{HF}$ & 16.0 & 19.6 & 13.4 \\
$\mathrm{MP} 2$ & 32.4 & 37.7 & 26.8 \\
$\mathrm{CCSD}(\mathrm{T})$ & 37.6 & 42.0 & 27.5 \\
$\mathrm{~B} 3 \mathrm{LYP}$ & 39.4 & 47.1 & 28.8 \\
$\mathrm{PBE} 0$ & 34.3 & 40.7 & 25.6 \\
$\mathrm{PBE}$ & 57.4 & 67.1 & 38.9 \\
$\mathrm{Zr} 8 \mathrm{O}_{16}$ & & & \\
$\mathrm{HF}$ & 35.7 & 25.1 & 20.5 \\
$\mathrm{MP} 2$ & 87.7 & 56.2 & 40.5 \\
$\mathrm{~B} 3 \mathrm{LYP}$ & 104.7 & 61.1 & 39.4 \\
$\mathrm{PBE} 0$ & 90.4 & 53.2 & 36.1 \\
$\mathrm{PBE}$ & 166.9 & 85.3 & 51.7 \\
$\mathrm{Zr} \mathrm{H}_{15} \mathrm{O}_{30}$ & & & \\
$\mathrm{HF}$ & 49.5 & 34.3 & 50.7 \\
$\mathrm{MP} 2$ & 106.4 & 69.3 & 106.7 \\
$\mathrm{~B} 3 \mathrm{LYP}$ & 104.3 & 73.1 & 127.8 \\
$\mathrm{PBE} 0$ & 94.1 & 62.7 & 112.7 \\
$\mathrm{PBE}$ & 193.1 & 99.2 & 208.2 \\
\hline & & &
\end{tabular}


Concerning the computed optical nonlinearities (electronic contribution), the largest responses are delivered by the pure PBE functional which overshoots both $\chi_{i i i i}^{(3)}$ and $\chi_{i i j j}^{(3)}$ values by a factor of five and two, respectively, as compared to the PBE0 and B3LYP functionals. The observed method dependence is in accord with the results listed in Table 4 which summarizes the second dipole hyperpolarizability axial tensorial components of $\mathrm{Zr}_{4} \mathrm{O}_{8} \mathrm{Zr}_{8} \mathrm{O}_{16}$ and $\mathrm{Zr}_{15} \mathrm{O}_{30}$ (Fig 7) computed at the HF, MP2 CCSD(T) B3LYP, PBE0 and PBE levels with a sufficiently flexible basis set that is expected to provide a reliable description of dynamic electron correlation effects at the MP2 and $\operatorname{CCSD}(\mathrm{T})$ levels of theory. Out of the three functionals considered here, B3LYP and PBE0 delivered values close to those obtained at $\operatorname{CCSD}(\mathrm{T})$ and MP2 levels. On the other hand, the pure PBE functional yielded largely overestimated microscopic second hyperpolarizabilities for either cluster size. Finally, to verify our qualitative estimation about the frequency dispersion effects reported in the main text, we studied the dynamic microscopic hyperpolarizabilities of $\mathrm{Zr}_{4} \mathrm{O}_{8}$ and $\mathrm{Zr}_{8} \mathrm{O}_{16}$ clusters at the time dependent PBE0 level of theory (TD-PBE0) using the widely applied sum-over-state (SOS) treatment developed by Orr and Ward [42], [58]. At this level of theory, that showed a good performance in the calculation of cluster static hyperpolarizabilities, the computed optical gaps of $\mathrm{Zr}_{4} \mathrm{O}_{8}$ and $\mathrm{Zr}_{8} \mathrm{O}_{16}$ (4.6 and $5.3 \mathrm{eV}$ respectively) are comparable to the vertical bandgap of the bulk. The outcomes of our SOS computations obtained with the jorge-DZP all-electron [59] basis set over the first 100 singlet excited states showed that the IDRI dynamic average second hyperpolarizability $\bar{\gamma}(-\omega ;, \omega,-\omega, \omega)(\omega=1550 \mathrm{~nm})$ should lie 11 and $10 \%$ higher than the static values of $\mathrm{Zr}_{4} \mathrm{O}_{8}$ and $\mathrm{Zr}_{8} \mathrm{O}_{16}$, respectively.

\section{Funding}

This project has received funding from the European Research Council (ERC) under the European Union's Horizon 2020 research and innovation program (ERC POPSTAR - Grant Agreement No. 647342), the French Industry Ministry Nano2017 program and the ANR (Agence Nationale de la Recherche) FOIST. The authors also acknowledge C2N Nanocenter of the French RENATECH network where the devices were fabricated, ANR "Investissement d'Avenir" program (10-EQPX-0050) for having funded the acquisition of the NANOTEM platform including a Dual-beam FIB-FEG FEI SCIOS system used in this work. Part of this work was granted access to the HPC resources of [CCRT/CINES/IDRIS] under the allocation 2018-2019 and 2019-2020 [A0040807031] made by GENCI (Grand Equipement National de Calcul Intensif). We also acknowledge the "Direction du Numérique" of the Université de Pau et des Pays de l'Adour for the computing facilities provided.

Conflict of Interest: The authors declare no conflict of interest.

\section{References}

1. H. Rong, R. Jones, A. Liu, O. Cohen, D. Hak, A. Fang, and M. Paniccia, "A continuous-wave raman silicon laser," Nature 433, 725 (2005).

2. E. A. Kittlaus, H. Shin, and P. T. Rakich, "Large brillouin amplification in silicon," Nat. Photonics 10, 463 (2016).

3. M. A. Foster, A. C. Turner, J. E. Sharping, B. S. Schmidt, M. Lipson, and A. L. Gaeta, "Broad-band optical parametric gain on a silicon photonic chip," Nature 441, 960 (2006).

4. D. J. Moss, R. Morandotti, A. L. Gaeta, and M. Lipson, "New cmos-compatible platforms based on silicon nitride and hydex for nonlinear optics," Nat. photonics 7, 597 (2013).

5. K. Narayanan and S. F. Preble, "Optical nonlinearities in hydrogenated-amorphous silicon waveguides," Opt. Express 18, 8998-9005 (2010).

6. S. Dai, F. Chen, Y. Xu, Z. Xu, X. Shen, T. Xu, R. Wang, and W. Ji, "Mid-infrared optical nonlinearities of chalcogenide glasses in ge-sb-se ternary system," Opt. express 23, 1300-1307 (2015).

7. C. Thu, P. Ehrenreich, K. K. Wong, E. Zimmermann, J. Dorman, W. Wang, A. Fakharuddin, M. Putnik, C. Drivas, A. Koutsoubelitis, M. Vasilopoulou, L. C. Palilis, S. Kennou, J. Kalb, T. Pfadler, and L. Schmidt-Mende, "Role of the Metal-Oxide Work Function on Photocurrent Generation in Hybrid Solar Cells," Sci. Reports 8, 3559 (2018).

8. C. López-Gándara, F. M. Ramos, and A. Cirera, "YSZ-Based Oxygen Sensors and the Use of Nanomaterials: A Review from Classical Models to Current Trends,” J. Sensors (2009). 
9. R. Ramesh and D. G. Schlom, "Whither Oxide Electronics?” MRS Bull. 33, 1006-1014 (2008).

10. M. Bazzan and C. Sada, "Optical waveguides in lithium niobate: Recent developments and applications," Appl. Phys. Rev. 2, 040603 (2015).

11. L. Mechin, "YBCO superconducting microbolometers fabricated by silicon micromachining," Ph.D. thesis (1996).

12. X. D. Wu, R. E. Muenchausen, N. S. Nogar, A. Pique, R. Edwards, B. Wilkens, T. S. Ravi, D. M. Hwang, and C. Y. Chen, "Epitaxial yttria-stabilized zirconia on (1102) sapphire for YBa2Cu3O7- $\delta$ thin films," Appl. Phys. Lett. 58, 304-306 (1991).

13. L. F. Chen, P. F. Chen, L. Li, S. L. Li, X. N. Jing, S. J. Pan, and Y. H. Guo, " $\mathrm{YBa}_{2} \mathrm{Cu}_{3} \mathrm{O}_{7}$ thin films grown on sapphire with epitaxial yttriaâĂ ̌̌stabilized zirconia buffer layers," Appl. Phys. Lett. 61, 2412-2413 (1992).

14. C. Jorel, H. Colder, A. Galdi, and L. Méchin, "Epitaxial PZT thin films on YSZ-buffered Si (001) substrates for piezoelectric MEMS or NEMS applications," IOP Conf. Series: Mater. Sci. Eng. 41, 012012 (2012).

15. T. Falcade and C. de Fraga Malfatti, "Fuel Cell: A Review and a New Approach About YSZ Solid Oxide Electrolyte Deposition Direct on LSM Porous Substrate by Spray Pyrolysis," in Electrochemical Cells-New Advances in Fundamental Researches and Applications, (InTech, 2012).

16. S. Heiroth, R. Ghisleni, T. Lippert, J. Michler, and A. Wokaun, "Optical and mechanical properties of amorphous and crystalline yttria-stabilized zirconia thin films prepared by pulsed laser deposition," Acta Materialia 59, 2330-2340 (2011).

17. X. Song, Z. Liu, M. Kong, C. Lin, L. Huang, X. Zheng, and Y. Zeng, "Thermal stability of yttria-stabilized zirconia (YSZ) and $\mathrm{YSZ} \mathrm{Al}_{2} \mathrm{O}_{3}$ coatings," Ceram. Int. 43, 14321-14325 (2017).

18. M. F. Manna, D. E. Grandstaff, G. C. Ulmer, and E. P. Vicenzi, "The Chemical Durability of Yttria-Stabilized ZrO2 pH and O2 Geothermal Sensors," Proc. Tenth Int. Symp. on Water Rock Interact. pp. 295-299 (2001).

19. S. K. Pandey, O. P. Thakur, R. Raman, A. Goyal, and A. Gupta, "Structural and optical properties of YSZ thin films grown by PLD technique," Appl. Surf. Sci. 257, 6833-6836 (2011).

20. R. C. Buchanan and S. Pope, "Optical and Electrical Properties of Yttria Stabilized Zirconia (YSZ) Crystals," J. The Electrochem. Soc. 130, 962-966 (1983).

21. N. Nicoloso, A. Löbert, and B. Leibold, "Optical absorption studies of tetragonal and cubic thin-film yttria-stabilized zirconia," Sensors Actuators B: Chem. 8, 253-256 (1992).

22. G. Marcaud, S. Matzen, C. Alonso-Ramos, X. Le Roux, M. Berciano, T. Maroutian, G. Agnus, P. Aubert, L. Largeau, V. Pillard, S. Serna, D. Benedikovic, C. Pendenque, E. Cassan, D. Marris-Morini, P. Lecoeur, and L. Vivien, "High-quality crystalline yttria-stabilized-zirconia thin layer for photonic applications," Phys. Rev. Mater. 2 (2018).

23. K. K. Gopalan, D. Rodrigo, B. Paulillo, K. K. Soni, and V. Pruneri, "Ultrathin Yttria-Stabilized Zirconia as a Flexible and Stable Substrate for Infrared Nano-Optics," Adv. Opt. Mater. 7, 966 (2019).

24. S. Serna and N. Dubreuil, "Bi-directional top-hat D-Scan: single beam accurate characterization of nonlinear waveguides," Opt. Lett. 42, 3072-3075 (2017).

25. M. A. Parkes, K. Refson, M. D' Avezac, G. J. Offer, N. P. Brandon, and N. M. Harrison, "Chemical Descriptors of Yttria-Stabilized Zirconia at Low Defect Concentration: An ab Initio Study,” J. Phys. Chem. A 119, 6412-6420 (2015).

26. M. A. Parkes, D. A. Tompsett, M. d'Avezac, G. J. Offer, N. P. Brandon, and N. M. Harrison, "The atomistic structure of yttria stabilised zirconia at 6.7 mol\%: an ab initio study," Phys. Chem. Chem. Phys. 18, 31277-31285 (2016).

27. D. M. Bishop, "Molecular vibrational and rotational motion in static and dynamic electric fields," Rev. Mod. Phys. 62, 343-374 (1990).

28. P. Karamanis, R. Marchal, P. Carbonnière, and C. Pouchan, "Doping-enhanced hyperpolarizabilities of silicon clusters: A global ab initio and density functional theory study of $\mathrm{Si}_{10}(\mathrm{Li}, \mathrm{Na}, \mathrm{K})_{n}(\mathrm{n}=1,2)$ clusters," The J. Chem. Phys. 135, 044511 (2011).

29. P. Karamanis, C. Pouchan, C. A. Weatherford, and G. L. Gutsev, "Evolution of Properties in Prolate (GaAs) $n$ Clusters," J. Phys. Chem. C 115, 97-107 (2011).

30. P. Karamanis, "The Importance of the DFT method on the computation of the second hyperpolarizability of semiconductor clusters of increasing size: A critical analysis on prolate aluminum phosphide clusters," Int. J. Quantum Chem. 112, 2115-2125 (2012).

31. J. P. Perdew, K. Burke, and M. Ernzerhof, “Generalized gradient approximation made simple," Phys. Rev. Lett. 77, 3865-3868 (1996).

32. C. Adamo and V. Barone, "Toward reliable density functional methods without adjustable parameters: The PBE0 model," J. Chem. Phys. 110, 6158-6170 (1999).

33. A. D. Becke, "Density-functional thermochemistry. III. The role of exact exchange," J. Chem. Phys. 98, 5648-5652 (1993).

34. C. Ricca, A. Ringuedé, M. Cassir, C. Adamo, and F. Labat, "Revealing the properties of the cubic $\mathrm{ZrO}_{2}(111)$ surface by periodic DFT calculations: reducibility and stabilization through doping with aliovalent $\mathrm{Y}_{2} \mathrm{O}_{3}$," RSC Adv. 5, 13941 (2015).

35. R. Dovesi, A. Erba, R. Orlando, C. M. Zicovich-Wilson, B. Civalleri, L. Maschio, M. Ferrabone, M. Rérat, S. Casassa, J. Baima, S. Salustro, and B. Kirtman, "Quantum-mechanical condensed matter simulations with crystal," WIREs Comput. Mol. Sci. 8, e1360 (2018).

36. M. J. Frisch, G. W. Trucks, H. B. Schlegel, G. E. Scuseria, M. A. Robb, J. R. Cheeseman, G. Scalmani, V. Barone, B. Mennucci, G. A. Petersson, H. Nakatsuji, M. Caricato, X. Li, H. P. Hratchian, A. F. Izmaylov, J. Bloino, G. Zheng, 
J. L. Sonnenberg, M. Hada, M. Ehara, K. Toyota, R. Fukuda, J. Hasegawa, M. Ishida, T. Nakajima, Y. Honda, O. Kitao, H. Nakai, T. Vreven, J. A. Montgomery, Jr., J. E. Peralta, F. Ogliaro, M. Bearpark, J. J. Heyd, E. Brothers, K. N. Kudin, V. N. Staroverov, R. Kobayashi, J. Normand, K. Raghavachari, A. Rendell, J. C. Burant, S. S. Iyengar, J. Tomasi, M. Cossi, N. Rega, J. M. Millam, M. Klene, J. E. Knox, J. B. Cross, V. Bakken, C. Adamo, J. Jaramillo, R. Gomperts, R. E. Stratmann, O. Yazyev, A. J. Austin, R. Cammi, C. Pomelli, J. W. Ochterski, R. L. Martin, K. Morokuma, V. G. Zakrzewski, G. A. Voth, P. Salvador, J. J. Dannenberg, S. Dapprich, A. D. Daniels, O. Farkas, J. B. Foresman, J. V. Ortiz, J. Cioslowski, and D. J. Fox, "Gaussian09 Revision D.01," Gaussian Inc. Wallingford CT 2009.

37. E. V. Stefanovich, A. L. Shluger, and C. R. A. Catlow, "Theoretical study of the stabilization of cubic-phase $\mathrm{ZrO}_{2}$ by impurities," Phys. Rev. B 49, 11560-11571 (1994).

38. R. A. Ploc, "The lattice parameter of cubic $\mathrm{ZrO}_{2}$ formed on zirconium," J. Nucl. Mat. 99, 124-128 (1981).

39. E. Elizalde, J. M. Sanz, F. Yubero, and L. GalÃąn, "Determination of optical constants of $\mathrm{ZrO} 2$ and $\mathrm{Zr}$ by electron energy-loss spectroscopy,” Surf. Interface Analysis 16, 213-214 (1990).

40. D. W. McComb, "Bonding and electronic structure in zirconia pseudopolymorphs investigated by electron energy-loss spectroscopy,” Phys. Rev. B 54, 7094-7102 (1996).

41. D. L. Wood and K. Nassau, "Refractive index of cubic zirconia stabilized with yttria," Appl. Opt. 21, 2978-2981 (1982).

42. B. Orr and J. Ward, "Perturbation theory of the non-linear optical polarization of an isolated system," Mol. Phys. 20, 513-526 (1971).

43. S. J. A. van Gisbergen, P. R. T. Schipper, O. V. Gritsenko, E. J. Baerends, J. G. Snijders, B. Champagne, and B. Kirtman, "Electric Field Dependence of the Exchange-Correlation Potential in Molecular Chains," Phys. Rev. Lett. 83, 694-697 (1999).

44. L. Zibordi-Besse, Y. Seminovski, I. Rosalino, D. Guedes-Sobrinho, and J. L. F. Da Silva, "Physical and Chemical Properties of Unsupported $\left(\mathrm{MO}_{2}\right)_{n}$ Clusters for $\mathrm{M}=\mathrm{Ti}, \mathrm{Zr}$, or $\mathrm{Ce}$ and $n=1-15$ : A Density Functional Theory Study Combined with the Tree-Growth Scheme and Euclidean Similarity Distance Algorithm,” The J. Phys. Chem. C 122, 27702-27712 (2018).

45. J. Zaanen, G. A. Sawatzky, and J. W. Allen, "Band gaps and electronic structure of transition-metal compounds," Phys. Rev. Lett. 55, 418-421 (1985).

46. S. Serna, J. Oden, M. Hanna, C. Caer, X. L. Roux, C. Sauvan, P. Delaye, E. Cassan, and N. Dubreuil, "Enhanced nonlinear interaction in a microcavity under coherent excitation," Opt. Express 23, 29964-29977 (2015).

47. A. Major, F. Yoshino, I. Nikolakakos, J. S. Aitchison, and P. W. Smith, "Dispersion of the nonlinear refractive index in sapphire," Opt. letters 29, 602-604 (2004).

48. K. Ikeda, R. E. Saperstein, N. Alic, and Y. Fainman, "Thermal and Kerr nonlinear properties of plasma-deposited silicon nitride/ silicon dioxide waveguides," Opt. Express 16, 12987 (2008).

49. S. C. Pinault and M. Potasek, "Frequency broadening by self-phase modulation in optical fibers," JOSA B 2 , 1318-1319 (1985).

50. B. Champagne, E. A. Perpète, J.-M. André, and B. Kirtman, "Analysis of the vibrational static and dynamic second hyperpolarizabilities of polyacetylene chains," Synth. Met. 85, 1047-1050 (1997).

51. R. Dovesi, V. R. Saunders, C. Roetti, R. Orlando, C. M. Zicovich-Wilson, F. Pascale, B. Civalleri, K. Doll, N. M. Harrison, I. J. Bush, P. D’Arco, M. Llunell, M. Causà, Y. Noël, L. Maschio, A. Erba, M. Rérat, and S. Casassa, CRYSTAL17 User's Manual, Univ. Torino (2017).

52. G. Hurst, M. Dupuis, and E. Clementi, "Ab initio analytic polarizability, first and second hyperpolarizabilities of large conjugated organic molecules: Applications to polyenes $\mathrm{C}_{4} \mathrm{H}_{6}$ to $\mathrm{C}_{22} \mathrm{H}_{24}$," J. Chem. Phys. 89, 385 (1988).

53. M. Ferrero, M. Rérat, R. Orlando, and R. Dovesi, "The calculation of static polarizabilities of periodic compounds. The implementation in the CRYSTAL code for 1D, 2D and 3D systems," J. Comp. Chem. 29, 1450-1459 (2008).

54. M. Ferrero, M. Rérat, R. Orlando, and R. Dovesi, "Coupled perturbed Hartree-Fock for periodic systems: The role of symmetry and related computational aspects," J. Chem. Phys. 128, 014110 (2008).

55. R. Orlando, V. Lacivita, R. Bast, and K. Ruud, "Calculation of the first static hyperpolarizability tensor of threedimensional periodic compounds with a local basis set: A comparison of LDA, PBE, PBE0, B3LYP, and HF results," J. Chem. Phys. 132, 244106 (2010).

56. M. Ferrero, M. Rérat, B. Kirtman, and R. Dovesi, "Calculation of first and second static hyperpolarizabilities of oneto three-dimensional periodic compounds. Implementation in the CRYSTAL code," J. Chem. Phys. 129, 244110 (2008).

57. L. Valenzano, F. J. Torres, K. Doll, F. Pascale, C. M. Zicovich-Wilson, and R. Dovesi, "Ab Initio Study of the Vibrational Spectrum and Related Properties of Crystalline Compounds; the Case of CaCO3 Calcite," Zeitschrift für Physikalische Chemie 220, 893-912 (2006).

58. T. Lu and F. Chen, "Multiwfn: A multifunctional wavefunction analyzer," J. Comput. Chem. 33, 580-592 (2018).

59. K. L. Schuchardt, B. T. Didier, T. Elsethagen, L. Sun, V. Gurumoorthi, J. Chase, J. Li, and T. L. Windus, "Basis set exchange:âĂL a community database for computational sciences," J. Chem. Inf. Model. 47, 1045-1052 (2007). 01038 . 